\title{
Magnetotransport and nonadditivity of point-contact resistances in series
}

\author{
C. W. J. Beenakker \\ Philips Research Laboratories, Postbox 80.000, NL-5600 JA Eindhoven, The Netherlands \\ H. van Houten \\ Philips Laboratories, Briarcliff Manor, New York 10510
}

(Received 28 November 1988)

\begin{abstract}
A theoretical study is given of the resistance of two ballistic point contacts in series. Nonadditivity of resistances is demonstrated as a result of classical ballistic transport. A rich magnetoresistance behavior is predicted to follow from the interplay of the cyclotron radius, the pointcontact width, and the point-contact separation.
\end{abstract}

The fascination of ballistic transport in submicron structures in a two-dimensional electron gas (2D EG) derives in part from the fact that by tailoring the device geometry a wealth of new magnetotransport phenomena can be realized. ${ }^{1}$ These effects originate from the interplay of the various dimensions of the system with the cyclotron orbit radius. The basic building block for such devices is the ballistic point contact. Single point contacts have already shown a number of interesting magnetotransport effects, following the discovery of the conductance quantization in zero magnetic field. ${ }^{2,3}$ In a weak magnetic field the quantization can be used to determine directly the rate of depopulation of magnetoelectric subbands. ${ }^{3,4}$ A negative four-terminal magnetoresistance was found, ${ }^{5}$ and interpreted in terms of reduced backscattering by the point contact of magnetic edge states on the 2D EG boundary. An even richer magnetoresistance behavior is expected in a device which combines two point contacts. A striking example is the experiment on coherent electron focusing, ${ }^{6}$ in a geometry with two adjacent point contacts. In this paper we present a theoretical study of magnetotransport in a geometry with two opposite point contacts connected in series. This geometry is of particular interest because of the remarkable nonadditivity of point-contact resistances discovered experimentally by Wharam et al. ${ }^{7}$

We consider the geometry shown in Fig. 1 of two identical point contacts facing each other at opposite boundaries of the 2D EG (extension of our results to unequal point contacts is cumbersome, but straightforward). Our approach applies the Landauer-Büttiker formalism ${ }^{8}$ for calculating resistances by means of transmission probabilities (cf. also Büttiker's analysis of tunneling through a double-barrier structure ${ }^{9}$ ). The point contacts are connected by $2 D$ EG leads to source and drain reservoirs at chemical potentials $\mu_{s}$ and $\mu_{d}$. A current $I$ flows from source to drain. We make the assumption that inelastic scattering has equilibrated the 2D EG far to the left and right of the point contacts, at chemical potentials $\mu_{l}$ and $\mu_{r}$. We will return to this assumption of equilibration, which is crucial for what follows. Electrons incident on the source point contact can reach the drain reservoir either directly (with transmission probability $T_{d}$ ), or subsequent to equilibration at the left and right end of the $2 \mathrm{D}$
EG (Ref. 10) (which are reached with transmission probabilities $T_{l}$ and $T_{r}$, respectively). The normalization of these transmission probabilities is such that

$$
T_{d}+T_{l}+T_{r}=N_{s}-R_{s},
$$

where $N_{s}$ is the number of spin degenerate quantum channels (or transverse wave-guide modes) in the lead to a point contact, and $R_{s}$ is the probability for electrons to be scattered back into the source reservoir. The right-hand side of Eq. (1) is proportional to the two-terminal ${ }^{11}$ conductance $G$ of an individual point contact, which is approximately quantized ${ }^{2,3}$ in units of $2 e^{2} / h$,

$$
G=\left(2 e^{2} / h\right)\left(N_{s}-R_{s}\right) \approx\left(2 e^{2} / h\right) N .
$$

The integer $N$ is the number of quantum channels in the narrow point contact itself. The Büttiker formula, ${ }^{8}$

$$
(h / 2 e) I_{\alpha}=\left(N_{\alpha}-R_{\alpha}\right) \mu_{\alpha}-\sum_{\beta \neq \alpha} T_{\beta \rightarrow a} \mu_{\beta},
$$

relates the current $I_{\alpha}$ in lead $\alpha$ (with $N_{\alpha}$ quantum channels) to the chemical potentials $\mu_{\beta}$ of each of the reser-

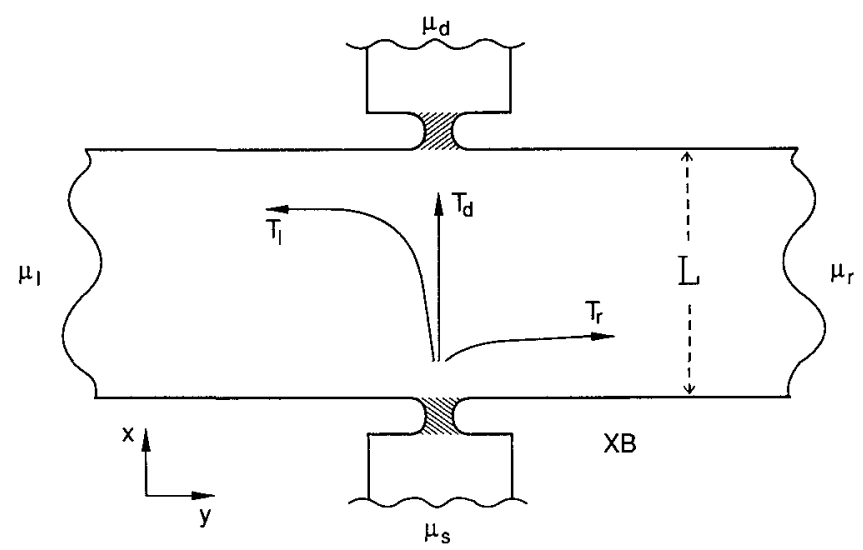

FIG. 1. Series point-contact geometry. Four reservoirs in local equilibrium at chemical potentials $\mu_{\alpha}$ and connected by impurity-free 2D EG regions are indicated schematically. As discussed in the text, local equilibrium at $\mu_{l}$ and $\mu_{r}$ may not be realized in a strong magnetic field. The shaded area in the point contacts indicates a possible reduced electron-gas density leading to a potential barrier. 
voirs, via transmission and reflection probabilities $T_{\beta \rightarrow a}$ (from reservoir $\beta$ to $\alpha$ ) and $R_{a}$ (from reservoir $\alpha$ back to the same reservoir). In our case $\alpha, \beta=s, d, r, l$. Since the full current $I$ must flow through the two point contacts, we require that $I_{r}=I_{l}=0$, and $I_{s}=-I_{d}=I$. Thus, one obtains a set of equations which (after some lengthy algebra and exploiting the symmetry of the geometry) yield the required expression for the series conductance $G_{\text {series }}$ $\equiv e I /\left(\mu_{s}-\mu_{d}\right)$,

$$
G_{\text {series }}=\frac{1}{2}\left[G+\frac{2 e^{2}}{h}\left(T_{d}+\frac{\left(T_{r}-T_{l}\right)^{2}}{2\left(N_{r}-R_{r}\right)-T_{r}-T_{l}}\right)\right] .
$$

We first consider the case of zero magnetic field. Then $T_{r}=T_{l}$, and we simply have

$$
G_{\text {series }}(B=0)=\frac{1}{2}\left[G+\left(2 e^{2} / h\right) T_{d}\right] .
$$

This formula is equivalent to that obtained by Büttiker for tunneling in series barriers. ${ }^{9}$ Ohmic addition of conductances is found if $T_{d}=0$ (then all transmission is with intervening equilibration). At the opposite extreme, if all transmission is direct, then $T_{d}=N_{s}-R_{s}=\left(h / 2 e^{2}\right) G$ by Eqs. (1) and (2), so that the series conductance is identical to that of the single point contact. The direct transmission probability (and thereby the non-Ohmic behavior) can be enhanced by collimation of the beam of injected electrons. One way of achieving this effect is by means of a potential barrier in the point contacts. The injected electrons then have velocity directions restricted to a cone of allowed angles $\phi$ with the $x$ axis defined by $E_{F} \cos ^{2} \phi$ $>E_{0}$ (with $E_{F}$ the Fermi energy and $E_{0}$ the barrier height). A second way is flaring the point contacts to form a horn. We assume that the horn widens gradually from a width $W_{\min }$ to $W_{\max }$, and treat the motion within the horn as adiabatic. ${ }^{12}$ The absolute value of the product of horn width and $y$ component of the velocity is an adiabatic invariant, so that the injected electrons now have velocity directions restricted to the cone $W_{\max } E_{F}^{1 / 2}|\sin \phi|$ $<W_{\min }\left(E_{F}-E_{0}\right)^{1 / 2}$. (Within this cone the angular distribution is $\propto \cos \phi$, the same as without collimation.) Diffraction of the electron beam inhibits its collimation for point contacts narrower than a wave length. For much wider contacts diffraction is unimportant, and in this case the transmission probabilities can be obtained from the classical trajectories. Note that $T_{d} / N$ is the fraction of the injected current in the segment of the beam which does not miss the opening of the opposite point contact. For $f W_{\max } \ll L$ we have found the simple formula

$$
G_{\text {series }}(B=0)=\frac{1}{2} G\left(1+f W_{\max } / 2 L\right),
$$

plotted in Fig. 2 (dashed lines). Here $L$ denotes the exitto-entrance separation of the two point contacts (see Fig. 1 ), and $f=f_{b} f_{h}$ is the product of a barrier collimation factor $f_{b} \equiv\left(1-E_{0} / E_{F}\right)^{-1 / 2}$ and a horn collimation factor $f_{h} \equiv W_{\max } / W_{\min }$. For wider contacts, $G_{\text {series }}$ can be written as a spatial integral over the contact opening. This expression is lengthy and we only show the results (solid curves in Fig. 2), which are quite close to the asymptotic formula (6) for $W_{\max } \lesssim L$ and moderate collimation.

We pause to discuss the experiment by Wharam et al., ${ }^{7}$

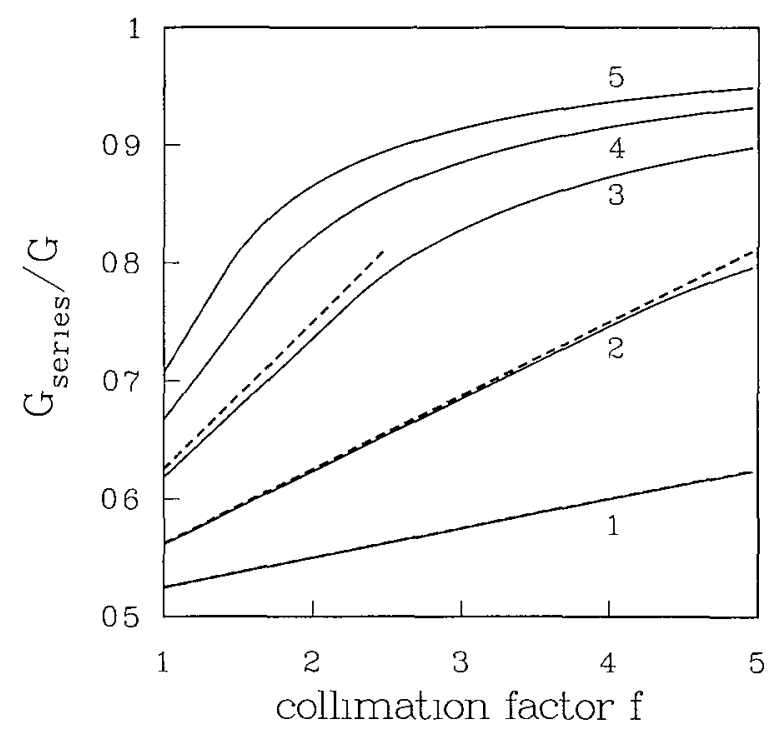

FIG. 2. Calculated classical series conductance at zero magnetic field as a function of the collimation factor defined in the text $\left(W_{\max } / L=0.1,0.25,0.5,0.75\right.$, and 1 for the curves numbered $1,2,3,4$, and 5 , respectively). The dashed lines are from the asymptotic formula (6).

which motivated the above analysis. Two opposite point contacts of variable width are defined electrostatically in a 2D EG, by means of two parallel split gates on top of a GaAs- $\mathrm{Al}_{x} \mathrm{Ga}_{1-x} \mathrm{As}$ heterostructure. The resistances of the point contacts are measured separately (by turning off the voltage on the gate for the other point contact), as well as in series. Our results in Fig. 2 and Eq. (6) are specific for classical point contacts, and do not, therefore, describe the plateaus in the series resistance as a function of gate voltage observed experimentally. The nonadditivity, however, is essentially a classical ballistic effect, and should, therefore, be reasonably well described by classical transmission probabilities. We consider the situation where both point contacts have the same conductance of $5 \times\left(2 e^{2} / h\right)$ (at a voltage of $-1 \mathrm{~V}$ on both gates). The (minimal) width of the point contacts is then estimated ${ }^{7}$ at $W_{\min } \approx 0.125 \mu \mathrm{m}$, and from the scale diagram (Fig. 2 in Ref. 7) we estimate an exit-to-entrance separation of $L \approx 0.7 \mu \mathrm{m}$. The width $W_{\max }$ at the exit and entrance of the point contacts is not known, but is not likely to be far from the width of the opening in the split gate, which is given as $0.3 \mu \mathrm{m}$. The barrier height $E_{0}$ is presumably small for this gate voltage at which the 2D EG directly under the gate has just been depleted, and we ignore it. In Fig. 3 of Ref. 7 it is shown that the series resistance of the two point contacts is higher than that of the single point contact by about a factor of 1.4 , which is considerably less than the factor of 2 expected from Ohmic addition. Substituting the estimated experimental parameters in Eq. (6) (or using Fig. 2 ) we find $G / G_{\text {seres }} \approx 1.3$, close to what is found experimentally.

We now turn to the effects of a magnetic field on the series conductance. The Lorentz force deflects the electrons, thus increasing $T_{r}$ at the expense of $T_{d}$ as well as $T_{l}$ (Fig. 1). These two effects compete, according to Eq. (4): Reduction of the nrobability for direct $\mathrm{t}^{-} \mathrm{ar}$ ni sion $T_{d}$ 
lowers $G_{\text {series, }}$ whereas reduction of $T_{l}$ relative to $T_{r}$ raises $G_{\text {series }}$ by increasing the probability for indirect transmission (with intervening equilibration). Figure 3 (inset) illustrates how these effects compete in the case of classical transmission probabilities [the calculation is not given here, but is similar to that leading to Eq. (6) with the Lorentz force deffection of the injected beam taken into account]. In the absence of collimation (dashed curve) the effect of $B$ on the indirect transmission donates, while with collimation (solid curve) the effect on the direct transmission takes over, causing a rapid drop of $G_{\text {series }}$ when the collimated beam sweeps past the drain point contact on increasing the magnetic field.

In a strong magnetic field, $T_{d}$ and $T_{l}$ become negligibly small compared to $T_{r}$, so that only indirect transmission remains. Note that $T_{d}=T_{l}=0$ implies via Eqs. (1) and (2) that $T_{r}=\left(h / 2 e^{2}\right) G$. The criterion for this field regime is $2 l_{c}<L$, with $l_{c} \equiv \hbar k_{F} / e B$ the cyclotron radius in the region between the point contacts $\left(k_{F}\right.$ is the Fermi wave vector in that region). From Eq. (4) we then find

$$
G_{\text {series }}=\left(2 G^{-1}-G_{H}^{-1}\right)^{-1},
$$

with $G_{H} \equiv\left(2 e^{2} / h\right)\left(N_{r}-R_{r}\right)$. In the strong-field regime backscattering is negligible ${ }^{13}$ in the region between the point contacts $\left(R_{r}=0\right)$, and moreover $N_{r}=N_{L}$ equals the number of occupied Landau levels in that region. The conductance $G_{H}$ is, therefore, simply the quantum Hall conductance. If $G$ is quantized according to Eq. (2), then we can write

$$
G_{\text {series }}=\frac{2 e^{2}}{h}\left(2 / N-1 / N_{L}\right)^{-1}
$$

The physical origin of the simple addition rule (7) is that a strong magnetic field decouples the chemical potential differences $\mu_{s}-\mu_{r}$ and $\mu_{r}-\mu_{d}$ (which are identical for

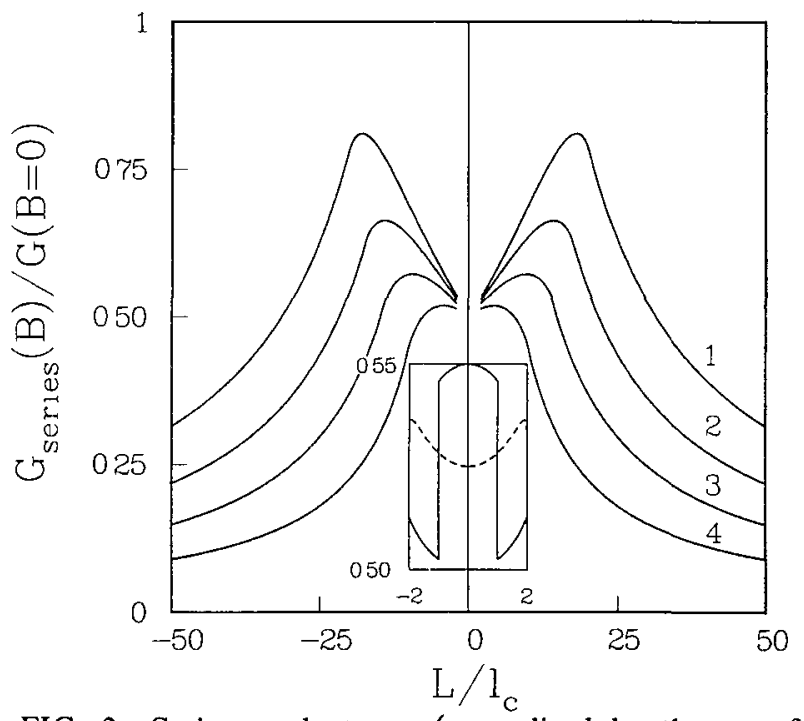

FIG. 3. Series conductance (normalized by the zero-field conductance of the individual point contacts) as a function of reduced magnetic field $\left(W_{\min }=W_{\max }=0.1 L\right.$, and $E_{0} / E_{F}=0,0.25$, 0.5 , and 0.75 for the curves numbered $1,2,3$, and 4 , respectively). The classical weak-field behavior is shown enlarged in the inset $\left(E_{0} / E_{F}=0\right.$ and 0.75 for the dashed and solid curves, respectively).
$B=0$ ). For $n$ different point contacts in series Eq. (7) generalizes to $G_{\text {series }}^{-1}-G_{H}^{-1}=\sum_{l=1}^{n} R_{4 t}(i)$, where $R_{4 t}(i)$ $\equiv G_{t}^{-1}-G_{H}^{-1}$ is the four-terminal longitudinal resistance $^{5,13}$ of a point contact with two-terminal conductance $G_{i}$.

Equation (7) predicts a curious camel-back shape for $G_{\text {series. }}$ This can most easily be seen by disregarding the discreteness of $N$ and $N_{L}$. We then have $N_{L}=\frac{1}{2} k_{F} l_{c}$; a formula for the magnetic field dependence of $N$ is given in Refs. 4 and 5. In Fig. 3 we show the resulting $B$ dependence of $G_{\text {series. }}$. The nonmonotonic behavior is due to the delayed depopulation of subbands in the point contacts, compared to the broad 2D EG. While the number of occupied Landau levels $N_{L}$ in the region between the point contacts decreases steadily with $B$ for $2 l_{c}<L$, the number $N$ of occupied subbands in the point contacts remains approximately constant ${ }^{4}$ until $2 l_{c, \min } \approx W_{\min }$ [with $l_{c, \min }$ $\equiv l_{c}\left(1-E_{0} / E_{F}\right)^{1 / 2}$ being the cyclotron radius in the point contact region]. In this field interval $G_{\text {series }}$ increases with $B$, according to Eq. (7). For stronger fields, depopulation in the point contacts begins to dominate $G_{\text {series, leading }}$ finally to a decreasing conductance (as is the rule for single point contacts ${ }^{3,4}$ ). The peak in $G_{\text {series, thus, occurs at }}$ $2 l_{c, \min } \approx W_{\text {min }}$. On increasing the barrier height in the point contacts the peak shifts to lower fields, becoming less pronounced (cf. Fig. 3).

We return to the equilibration condition on which our analysis is based. In a quantizing magnetic field, local equilibrium is reached by interLandau-level scattering. If the potential landscape (both in the point contacts themselves and in the $2 \mathrm{D}$ EG region in between) varies by less than the Landau level separation $\hbar \omega_{c}\left(\omega_{c} \equiv e B / m\right)$ on the length scale of the magnetic length $(\hbar / e B)^{1 / 2}$, then interLandau-level scattering is suppressed in the absence of other scattering mechanisms. This means that the transport from one point contact to the other is adiabatic. The series conductance is then simply $G_{\text {series }}=\left(2 e^{2} / h\right) N$ for two identical point contacts $\left[N \equiv \min \left(N_{1}, N_{2}\right)\right.$ for two different point contacts in series]. This expression differs from Eq. (7) if a barrier is present in the point contacts, since that causes the number $N$ of occupied Landau levels in the point contact to be less than the number $N_{L}$ of occupied levels in the bulk 2D EG. IIn a strong magnetic field $N \approx\left(E_{F}-E_{0}\right) / \hbar \omega_{c}$, while $N_{L} \approx E_{F} / \hbar \omega_{c}$.] Adiabatic transport between two adjacent point contacts has been demonstrated experimentally in Ref. 14. We emphasize that in the geometry of Fig. 1 a strong magnetic field is necessary for adiabatic transport. ${ }^{15}$ In zero field the quantum number increases greatly when the electron leaves the narrow point contact to enter the broad region (this mode conversion is treated theoretically in Ref. 16).

In summary, a theory has been presented for ballistic transport through two point contacts in series. Large deviations from Ohmic addition of resistances are found, in qualitative agreement with available experimental data. ${ }^{7}$ A rich magnetoresistance behavior is predicted. It would be of interest to look for these effects experimentally. By a suitable combination of measurements on the individual point contacts and on the composite structure it should be possible to determine the relevant parameters, so that a quantitative analysis becomes feasible. 
'See, for example, Physics and Technology of Submicron Structures, edited by H. Heinrich, G. Bauer, and F. Kuchar, Springer Series in Solid State Sciences, Vol. 83 (SpringerVerlag, Berlin, 1988).

${ }^{2}$ B. J. van Wees, H. van Houten, C. W. J. Beenakker, J. G. Williamson, L. P. Kouwenhoven, D. van der Marel, and C. T. Foxon, Phys. Rev. Lett. 60, 848 (1988).

${ }^{3}$ D. A. Wharam, T. J. Thornton, R. Newbury, M. Pepper, H. Ahmed, J. E. F. Frost, D. G. Hasko, D. C. Peacock, D. A. Ritchie, and G. A. C. Jones, J. Phys. C 21, L209 (1988).

${ }^{4}$ B. J. van Wees, L. P. Kouwenhoven, H. van Houten, C. W. J. Beenakker, J. E. Mooij, C. T. Foxon, and J. J. Harris, Phys. Rev. B 38, 3625 (1988).

${ }^{5}$ H. van Houten, C. W. J. Beenakker, P. H. M. van Loosdrecht, T. J. Thornton, H. Ahmed, M. Pepper, C. T. Foxon, and J. J. Harris, Phys. Rev. B 37, 8534 (1988).

${ }^{6}$ H. van Houten, B. J. van Wees, J. E. Mooij, C. W. J. Beenakker, J. G. Williamson, and C. T. Foxon, Europhys. Lett. 5, 721 (1988); C. W. J. Beenakker, H. van Houten, and B. J. van Wees, ibid. 7, 359 (1988).

${ }^{7}$ D. A. Wharam, M. Pepper, H. Ahmed, J. E. F. Frost, D. G. Hasko, D. C. Peacock, D. A. Ritchie, and G. A. C. Jones, J. Phys. C 21, L887 (1988).

${ }^{8}$ M. Büttiker, Phys. Rev. Lett. 57, 1761 (1986); IBM J. Res. Dev. 32, 317 (1988).

${ }^{9}$ M. Büttiker, Phys. Rev. B 33, 3020 (1986); IBM J. Res. Dev. 32, 63 (1988).

${ }^{10}$ The indirect contribution can be eliminated by operating one of the point contacts as a voltage probe (drawing no net current), as is done in the electron focusing experiment (Ref.
6). This is an interesting configuration to detect the electron beam injected by a point contact, but will not be discussed here any further.

${ }^{11}$ The two-terminal conductance of an individual point contact is defined as $G \equiv e I / \mu_{s}$, with the constraint $\mu_{l} \equiv \mu_{r} \equiv \mu_{d} \equiv 0$ (i.e., these chemical potentials are kept at a common ground potential). We also note that the series conductance $G_{\text {series }}$ is $1 / R_{s d, s d}$ in Büttiker's notation (Ref. 8). To obtain our expression for $G_{\text {series }}$ [Eq. (4)] from the general four-terminal resistance formula in Ref. 8 , one needs to identify $T_{a \rightarrow a} \equiv R_{a}$ in that formula.

${ }^{12}$ Conductance quantization as a result of adiabatic transport through a point contact has been studied by L. I. Glazman, G. B. Lesovik, D. E. Khmel'nitskii, and R. I. Shekhter, Pis'ma Zh. Eksp. Teor. Fiz. 48, 218 (1988) [JETP Lett. 48, 238 (1988)]. Flaring the point contact also reduces quantum mechanical reflections, as discussed in this context by $R$. Landauer, Z. Phys. B 68, 217 (1987).

${ }^{13}$ M. Büttiker, Phys. Rev. B 38, 9375 (1988).

${ }^{14}$ B. J. van Wees, E. M. M. Willems, C. J. P. M. Harmans, C. W. J. Beenakker, H. van Houten, J. G. Williamson, C. T. Foxon, and J. J. Harris, Phys. Rev. Lett. 62, 1181 (1989).

${ }^{15}$ Wharam et al. (Ref. 7), on the contrary, claim that the transport in their experiment without a magnetic field is determined by the conservation of quantum number. Their observation (Fig. 2 in their paper) of a $40 \%$ enhancement of the series resistance of two identical point contacts above the value of the individual resistances strongly suggests, however, that adiabatic transport was not experimentally realized.

${ }^{16}$ A. Szafer and A. D. Stone, Phys. Rev. Lett. 62, 300 (1989). 